\title{
Odd Triperfect Numbers
}

\section{By Masao Kishore*}

Abstract. We prove that an odd triperfect number has at least ten distinct prime factors.

1. A positive number $N$ is called a triperfect number if $\sigma(N)=3 N$, where $\sigma(N)$ is the sum of the positive divisors of $N$. Six even triperfect numbers are known:

$$
\begin{aligned}
& 2^{14} \cdot 5 \cdot 7 \cdot 19 \cdot 31 \cdot 151, \\
& 2^{13} \cdot 3 \cdot 11 \cdot 43 \cdot 127, \\
& 2^{9} \cdot 3 \cdot 11 \cdot 31, \\
& 2^{8} \cdot 5 \cdot 7 \cdot 19 \cdot 37 \cdot 73, \\
& 2^{5} \cdot 3 \cdot 7, \\
& 2^{3} \cdot 3 \cdot 5
\end{aligned}
$$

However, the existence of an odd triperfect (OT) number is an open question. McDaniel [1] and Cohen [2] proved that an OT number has at least nine distinct prime factors, and Beck and Najar [3] showed that it exceeds $10^{50}$.

In this paper using the technique of [4], we prove

THEOREM. If $N$ is $O T, N$ has at least ten distinct prime factors.

2. Throughout this paper we let

$$
N=\prod_{i=1}^{r} p_{i}^{a},
$$

where $p_{i}$ 's are odd primes, $p_{1}<\cdots<p_{r}$ and $a_{i}$ 's are positive integers.

The following lemmas are easy to prove:

LEMMA 1. If $N$ is $O T$,

$$
a_{i} \text { 's are even for } 1 \leqslant i \leqslant r .
$$

LEMMA 2. If $N$ is $O T$ and $q$ is a prime factor of $\sigma\left(p_{i}^{a_{i}}\right)$ for some $i$, then $q=3$ or $q=p_{j}$ for some $j, 1 \leqslant j \leqslant r$.

LEMMA 3. If $N$ is $O T$ and $r=9, p_{8}<80$.

Received July 6, 1981.

1980 Mathematics Subject Classification. Primary 10A20.

* This paper was written as a quarter project when the author was in a programming language class taught by Mr. Joseph Klinger at the University of Toledo in Spring, 1981. 
As in [4] we define

$$
\begin{aligned}
S(N) & =\sigma(N) / N, \\
a(p) & =\operatorname{Min}\left\{a \mid a \text { is even and } p^{a+1}>10^{11}\right\}, \\
b_{i} & =\operatorname{Min}\left\{a_{i}, a\left(p_{i}\right)\right\}, \\
M & =\prod_{i=1}^{r} p_{i}^{b_{1}} .
\end{aligned}
$$

LEMMA 4. If $N$ is $O T$, then

$$
\log 3-r \cdot 10^{-11}<\log S(M) \leqslant \log 3 .
$$

Proof. Since $M \mid N, S(M) \leqslant S(N)=3$ and so $\log S(M) \leqslant \log 3$. In [4] we proved that if $a>a(p)$, then

$$
0<\log S\left(p^{a}\right)-\log S\left(p^{a(p)}\right)<10^{-11} .
$$

Hence

$$
0<\log S(N)-\log S(M)<r \cdot 10^{-11} \text {, }
$$

and we have

$$
\log 3-r \cdot 10^{-11}=\log S(N)-r \cdot 10^{-11}<\log S(M) .
$$

Q.E.D.

COROLLARY. If $N$ is $O T, L=M / p_{r}^{b_{r}}$ and if $p_{r}>3500$, then

$$
\log 3-r \cdot 10^{-11}-\log S\left(3499^{2}\right)<\log S(L)<\log 3 \text {. }
$$

Proof of Theorem. We used a computer (PDP 11 at the University of Toledo) to find

$$
M=\prod_{i=1}^{9} p_{i}^{a_{i}}
$$

satisfying (1), $a_{i} \leqslant a\left(p_{i}\right)$ for $1 \leqslant i \leqslant 9$, (2) with $r=9$, and $p_{9}<3500$. There were 71 such $M$ 's; however, all of them had a factor $p_{i}^{a_{i}}$ such that $a_{i}<a\left(p_{i}\right), \sigma\left(p_{i}^{a_{i}}\right)$ had a prime factor $q>3$, and $q \neq p_{j}, 1 \leqslant j \leqslant 9$.

Next we tried to find

$$
L=\prod_{i=1}^{8} p_{i}^{a_{i}}
$$

satisfying (1), $a_{i} \leqslant a\left(p_{i}\right)$ for $1 \leqslant i \leqslant 8$, and (3) with $r=9$. There were 12689 such $L$ 's; however, 12473 of them had a factor $p_{i}^{a_{1}}$ such that $a_{i}<a\left(p_{i}\right), \sigma\left(p_{i}^{a_{i}}\right)$ had a prime factor $q>3, q \neq p_{j}$ for $1 \leqslant j \leqslant 8$, and

$$
\log S(L)+\log S\left(q^{2}\right)>\log 3 .
$$

The remaining 216 of them had the following properties: there exist two consecutive primes $u$ and $v$ such that $3500<u<v$,

$$
\begin{gathered}
\log S(L)+\log S\left(u^{2}\right)>\log 3, \text { and } \\
\log S(L)+\log v /(v-1)<\log 3-9 \cdot 10^{-11} .
\end{gathered}
$$


These three cases show that if $N$ is an odd integer with $r=9$, then $N$ cannot be OT. Q.E.D.

The computer time was over five hours.

Department of Mathematics

East Carolina University

Greenville, North Carolina 27834

1. W. McDaniel, “On odd multiply perfect numbers,” Boll. Un. Mat. Ital., No. 2, 1970, pp. 185-190.

2. G. L. COHEN, “On odd perfect numbers II, Multiperfect numbers and quasiperfect numbers," $J$. Austral. Math. Soc., v. 29, 1980, pp. 369-384.

3. W. E. Beck \& R. M. NajaR, “A lower bound for odd triperfects," Math. Comp., v. 38, 1982, pp. 249-251.

4. M. KISHORE, "Odd integers $N$ with five distinct prime factors for which $2-10^{-12}<\sigma(N) / N<2$ $+10^{-12}$," Math. Comp., v. 32, 1978, pp. 303-309. 